\title{
Wissenschaftliche Rationalität und verbrecherische Praxis
}

„Ostforschung"' als Förderschwerpunkt der DFG

(1920-1970)

Sabine Schleiermacher

Sören Flachowsky 2008: Von der Notgemeinschaft zum Reichsforschungsrat. Wissenschaftspolitik im Kontext von Autarkie, Aufrüstung und Krieg [= Studien zur Geschichte der Deutschen Forschungsgemeinschaft 3]. Stuttgart: Steiner, brosch., $545 \mathrm{~S}$., 60,00 €, ISBN-13: 978-3-51509-025-4.

Willi Oberkrome 2009: Ordnung und Autarkie. Die Geschichte der deutschen Landbauforschung, Agrarökonomie und ländlichen Sozialwissenschaft im Spiegel von Forschungsdienst und DFG (1920-1970) [= Studien zur Geschichte der Deutschen Forschungsgemeinschaft 4]. Stuttgart: Steiner, brosch., 371 S., 50,00 €, ISBN-13: 978-3-51509-255-5.

Karin Orth 2011: Autonomie und Planung der Forschung. Förderpolitische Strategien der Deutschen Forschungsgemeinschaft 1949-1968 [= Studien zur Geschichte der Deutschen Forschungsgemeinschaft 8]. Stuttgart: Steiner, brosch., 284 S., 45,00 €, ISBN-13: 978-3-515-09832-8.

Karin Orth und Willi Oberkrome (Hg.) 2010: Die Deutsche Forschungsgemeinschaft 1920-1970. Forschungsförderung im Spannungsfeld von Wissenschaft und Politik [= Beiträge zur Geschichte der Deutschen Forschungsgemeinschaft 4]. Stuttgart: Steiner, brosch., 549 S., 65,00 €, ISBN-13: 978-3-515-09652-2. 
Corinna R. Unger 2007: Ostforschung in Westdeutschland. Die Erforschung des europäischen Ostens und die Deutsche Forschungsgemeinschaft, 1945-1975 [= Studien zur Geschichte der Deutschen Forschungsgemeinschaft 1]. Stuttgart: Steiner, brosch., 497 S., 56,00 €, ISBN-13: 978-3-515-09026-1.

Karl Heinz Roth beschrieb 1993 die Geschichte der „Rezeption der ,Generalpläne Ost' der SS“ (Roth 1993), die in der deutschen Zeitgeschichtsforschung in der zweiten Hälfte der 1950er Jahre einsetzte. Bereits Mitte der 1980er Jahre hatte er auf die mangelnde Kenntnisnahme der Erforschung und Dokumentation nationalsozialistischer Verbrechen in den vom "Generalplan Ost" betroffenen Ländern durch die westdeutsche Wissenschaft hingewiesen, die er nicht nur in der sprachlichen Barriere begründet sah (Roth 1985: 70). Hatte Miroslav Kárný die Planungen 1977 schon als eine „erschütternde Verbindung von Wissenschaft und Verbrechen “ bezeichnet (Kárný 1977: 371, zitiert nach Eichholtz 1982: 224), so richtete sich das Forschungsinteresse nach der Dokumentation und ersten Analyse der verschiedenen Planungen und der an ihnen beteiligten Institutionen wie Personen auf die „enge und spezifische Verbindung von akademischer Forschung und nationalsozialistischer Planung, von Wissenschaft und ihrer Anwendung, von neuen theoretischen Ansätzen und rationaler Vernichtungspolitik" verschiedenster Disziplinen und wurde die „'Modernität' der Gesamtkonzepte für den Osten [...] vor allem in ihrer spezifisch wissenschaftlichen Rationalität" identifiziert (Rössler/ Schleiermacher 1993: 10). Seither hat sich die Forschung einzelnen Disziplinen und ihrer Beteiligung an der Ostraumplanung zugewendet. Die im Folgenden vorgestellten Publikationen untersuchen nun die Rolle der Deutschen Forschungsgemeinschaft (DFG) als Förderer der Forschungen zum "Generalplan Ost".

In den letzten Jahren erschienen Ergebnisse des Forschungsprojektes zur "Geschichte der Deutschen Forschungsgemeinschaft 1920-1970", das von ihrem damaligen Präsidenten, Ernst-Ludwig Winnacker, initiiert und unter der Leitung von Ulrich Herbert (Freiburg) und Rüdiger vom Bruch (Berlin) von 2000 bis 2008 durchgeführt wurde. ${ }^{1}$ Im Zentrum der Untersuchungen stand die Forschungspolitik der DFG während des Nationalsozialismus, die in den wissenschafts- und politikgeschichtlichen Kontext seit der Gründung der Vorläuferorganisation „Notgemeinschaft der Deutschen Wissenschaft" im Jahr 1920 (1929 in DFG umbenannt) eingeordnet wurde. Anhand institutionsgeschichtlicher Studien, vor allem aber mittels der Untersuchung einzelner, von der DFG initiierter Forschungsfelder und geförderter Projekte wurden die Forschungsaktivitäten selbst, ihre wissenschaftlichen Zielsetzungen, Biografien einzelner Wissenschaftler sowie die fächerübergreifende Forschungspolitik der DFG in den Blick genommen und in den Kontext nationaler wie internationaler Entwicklungen gestellt. In den exemplarischen Einzelstudien liegt das 
Augenmerk auf den Fragen nach dem Beziehungsgeflecht von Wissenschaft und Politik, den Motivationen und Handlungsspielräumen von Wissenschaftlern in der Hauptsache während des Nationalsozialismus sowie nach der Einordnung in die weitere Forschungslandschaft. Die Einzelstudien zeichnen dabei ein sich überschneidendes und ergänzendes Bild.

Auf die militärische Niederlage von 1918, den darauf folgenden Versailler Vertrag, vor allem aber die Festlegung neuer Grenzen im Osten, auf das Fortschreiten der Moderne, die Herausbildung von Parteien und Interessenverbänden mit der Aushandlung gegensätzlicher Interessen reagierte die akademische Elite des Deutschen Reiches, die um den Verlust ihres überproportionalen Einflusses in Politik und Gesellschaft fürchtete, mit radikalisiertem Nationalismus, der „nicht mehr als außerwissenschaftlicher Faktor, sondern als konstitutives Element einer spezifisch deutschen seriösen Wissenschaftlichkeit verstanden" wurde, und mit antiliberalen Überzeugungen und der Ablehnung der Weimarer Republik einherging (Wagner 2010a: 31). Im Mittelpunkt von Habitus, Mentalität und Grundorientierungen deutscher Ordinarien, „als deren Selbstverwaltung und Interessenvertretung die Notgemeinschaft und DFG in erster Linie agierten", identifiziert Sören Flachowsky für die erste Hälfte des 20. Jahrhunderts mit wenigen Ausnahmen „Autoritatismus [sic] der Gelehrten, ihre Vorbehalte gegenüber Parlamentarismus, Liberalismus und Demokratie sowie gegenüber Sozialisten und Juden“ (Flachowsky 2008: 48).

Vor dem Hintergrund sich ausweitender Forschungsaktivitäten in der Industrie und angesichts stagnierender absoluter finanzieller Mittel an den Universitäten sowie der allgemeinen Wirtschaftskrise der Nachkriegszeit hatten die Gelehrten aus Furcht vor dem Bedeutungsverlust der Wissenschaft und damit auch ihres eigenen Einflusses und Status den Zusammenschluss zur Notgemeinschaft betrieben.

Erfüllt vom Willen zur Revision des Versailler Vertrages sahen jene Kreise in der Wissenschaft das Potential, dem Deutschen Reich erneut zu großer Machtfülle zu verhelfen und mit dem Ausland wieder in Konkurrenz treten zu können. Es entstand eine zunehmend aggressive Haltung insbesondere gegenüber dem östlichen Europa, die von breiten Teilen der Gesellschaft geteilt wurde, so auch von Wissenschaftlern. Diese Grundüberzeugung, die als nationalistisch, deutschtumszentriert, völkisch und zunehmend biologistisch beschrieben werden kann, bestimmte das Verhalten von Wissenschaftlern über die Systemwechsel hinweg.

Die DFG erschien als „Reservat der Ordinarien“ und ihre Geschichte kann als „Geschichte des deutschen Professors“ verstanden werden. Die hier versammelten Wissenschaftler, durch Habitus, Mentalität und gemeinsame Interessen unterschiedlich miteinander verbunden, verstanden sich als „Elite mit Vertretungsanspruch für die Wissenschaft schlechthin“. Ihre wissenschaftliche Praxis war gekennzeichnet durch gegenseitige Begutachtung, 
Akkreditierung und eine flexibel dynamische „Anpassung an äußere wie innerwissenschaftliche Veränderung" (Wagner 2010a: 23-25). Während sie im Rahmen der DFG der 1920er bis 1940er Jahre mit hohem Engagement anwendungsorientierte Projekte vorantrieben, verstanden sie sich als Grundlagenforscher. Die Grenzziehung zwischen diesen Bereichen war eher symbolischer Natur und diente nach 1945 der Verschleierung der Beteiligung an der planerischen Vorbereitung nationalsozialistischer Verbrechen (Heinemann 2006: 66, Rössler 1990: 219-222).

Wichtiges Mittel der Forschungsförderung der Notgemeinschaft/DFG waren die interdisziplinär ausgerichteten, programmatische Schwerpunkte setzenden, praxis- und anwendungsbezogenen und als reichsweite Plattformen des Wissenstransfers dienenden Schwerpunktprogramme in der Form von „Gemeinschaftsarbeiten“. Sie entstanden als Bündelung exzellenter Forschungsvorhaben, vor dem Hintergrund alter Netzwerke, deren Strukturen bereits im Ersten Weltkrieg gelegt worden waren. Diese Netzwerke sorgten nicht nur für eine Mobilisierung der Wissenschaften, sondern auch für ihre enge Verbindung mit dem Militär und führten zu einer Enthemmung ihres Verhältnises zur modernen Kriegsführung und langfristig zu einem Heranrücken an staatliche und militärische Instanzen.

Mit der Förderung der sich noch formierenden Rassenforschung als Gemeinschaftsarbeit ab 1928 half die DFG bei der Modellierung eines neuen paradigmatischen Wissens-, Ordnungs- und Legitimationssystems. Gleiche Zuwendung, ab 1926 als Gemeinschaftsarbeit, erhielt die Ostforschung.

Die deutsche Ostforschung entstand als eigene Forschungsrichtung vor dem Hintergrund der Kriegsniederlage 1918, basierte auf tradierten antislawischen Ressentiments, die durch den entstehenden Antibolschewismus befördert wurden, und konnte durch diese Gemengelage begünstigt die bestehende Russlandforschung schnell überholen. Als an den Rändern unscharfes Konglomerat verschiedener Disziplinen formte sie sich als nationalistisches Metier, das seine Dynamik wie sein Erkenntnisinteresse aus Besitz- und Überlegenheitsansprüchen gegenüber Ostmittel- und Osteuropa generierte. Die DFG half mit ihrem Renommee wie mit ihren Finanzen der Ostforschung, sich in der wissenschaftlichen Gemeinschaft und ihren Institutionen zu etablieren und einen disziplinären Rahmen zu verschaffen. Seit der nationalsozialistischen Machteroberung wurde sie mit einer sprunghaft ansteigenden Fördersumme bedacht, während die DFG weiterhin bemüht war, ausschließliche Propagandaarbeit zumindest nicht finanziell zu fördern. Auch in diesem Forschungsfeld ist die politische, ideologische und wissenschaftliche Selbstmobilisierung der Wissenschaftler abzulesen. Corinna Unger kommt zu dem Ergebnis: 
Die erforderlichen intellektuellen, politischen und mentalen Dispositionen waren im rechtsnationalistischen Milieu, dem die meisten Vertreter der Ostforschung angehörten, seit den zwanziger Jahren vorhanden. [...] Die Ostforschung und die Ostforscher standen dem Nationalsozialismus nicht näher oder ferner, sondern sie waren Teil des Nationalsozialismus. (Unger 2007: 80)

Ein wichtiger Einschnitt nach der nationalsozialistischen Machteroberung war die Gründung des Reichsforschungsrates (RFR) im Jahr 1937, der für die Natur-, Agrar-, Sozial- und Technikwissenschaften zuständig war. Die DFG wandelte sich zur Verwaltungsstelle des RFR und Förderorganisation der Geisteswissenschaften. Die Satzungsänderung im Jahr 1938 beendete formal die partizipatorische Entscheidungsfindung im Kreise der Gelehrten und setzte auch in der DFG das „Führerprinzip“ um.

Ein Generationswechsel Ende der 1930er Jahre brachte junge, stark politisierte, pragmatisch orientierte und auf Effizienz bedachte Wissenschaftler, die zum Teil eng miteinander verbunden waren, in die Führungsspitze von DFG und RFR, mit denen sich die ältere Generation über konkurrierende Fraktionen, Seilschaften und Wissenschaftlertypen hinweg in dem Ziel verbunden wusste, „einen effektiven Beitrag zur Kriegsführung der Nation zu leisten“ (Wagner 2010a: 32). Für DFG und RFR gehörten die Forschungen zum „Generalplan Ost“ zu den mit den meisten Mitteln geförderten Projekten (Flachowsky 2008: 328 f.).

Die Reorganisation des RFR Ende 1942 hatte die Fokussierung auf unmittelbar kriegs- und rüstungsrelevante Forschungen im Rahmen der Autarkiebestrebungen und der nationalsozialistischen „Wehrwirtschaft" zum Ziel und machte ihn zur zentralen Finanzierungsstelle für jene Forschungsprojekte, die für die Expansionsziele des nationalsozialistischen Regimes essenziell waren, wie etwa die Begleitforschungen zum "Generalplan Ost". Dabei handelte es sich um sämtliche Planungen der verschiedensten Disziplinen bezüglich der gewünschten totalen Neugestaltung eines nationalsozialistischen Europas, insbesondere Osteuropas, die die „rassische“ Sortierung, Vertreibung und Umsiedlung von Millionen Menschen bedeutete (Flachowsky 2008: 330, 487, Oberkrome 2009: 216-218, Oberkrome 2010: 429, Herbert 2010: 494 f.).

Die Ermordung der europäischen Juden [bildete] gewissermaßen die Grundvoraussetzung [...]. Die Experten kalkulierten nämlich, dass im Planungszeitraum von 20 bis 30 Jahren das ,Judenproblem längst gelöst' wäre und die Juden mithin keine Siedlungsgebiete mehr benötigen würden. (Heinemann 2006: 53, Roth 1993: 33)

Insgesamt führte der Systemwechsel von 1933 zu einer Radikalisierung, die etwa die Kategorie „Volk“ als Leitbegriff für die Bewilligung von Projekten bei der DFG und Fluchtpunkt wissenschaftlichen Handelns noch weiter ins Zentrum stellte, durch die Verbindung mit „Rasse“ quasi naturwissenschaftlich auflud und schließlich den Pluralismus in der Forschung reduzierte. Dies 
führte zu einer Absage an den Weimarer Staat in den Grenzen von 1919 und der Hinwendung zu „Volksgrenzen“ und damit zur vermeintlich wissenschaftlich begründeten Forderung nach der Revision bestehender Grenzen und schließlich zur Unterstützung nationalsozialistischer Expansionsbestrebungen. Darüber hinaus ermöglichte er die Ausformung und Aufwertung einzelner Disziplinen, wie etwa der Geographie, Ethnologie, Linguistik und Geschichtswissenschaften.

Hiervon profitierte auch die Agrarforschung, die bereits vor 1933 von der DFG als Gemeinschaftsarbeit gefördert worden war. Mittels der in ihr zusammengefassten Disziplinen sollte auf der Basis einer wissenschaftlich fundierten Raumordnung sowie soziologischer Begleitforschung eine „Neubildung des deutschen Bauerntums" und "Germanisierung" Osteuropas vorangetrieben werden (Oberkrome 2009: 317). Unter der Parole „Volk und Nahrungsfreiheit" beschäftigten sich international renommierte Wissenschaftler verschiedener Fachrichtungen in dem von der DFG großzügig finanzierten Forschungsbereich mit Themen wie Industrie- und Siedlungsstrukturen, Bevölkerungsentwicklung, Technisierung und Rationalisierung von Arbeitsabläufen in der Landwirtschaft, Betriebswirtschaft, Züchtungsforschung sowie landwirtschaftliche Ertragssteigerung. Die Suche nach Ersatzstoffen für die Feldbestellung und Viehfutter sowie ihre Herstellung und Optimierung gehören in den Kontext autarkiewirtschaftlicher Fragestellungen und damit der kriegsrelevanten Forschung. Im Zusammenhang mit dem „Generalplan Ost“ und mit der Perspektive auf die „ultimative ,Eindeutschung' des Ostens" leisteten diese Forschungen mit ihren Expertisen Deportation und Umsiedlung Vorschub (Oberkrome 2010: 429).

Einzelnen Geisteswissenschaften fiel dabei die Rolle zu,

deutsche Dominanzansprüche in Europa mit historischen, linguistischen, volksund kulturkundlichen Studien zu untermauern und das geistige Profil potentieller Kriegsgegner wissenschaftlich zu vermessen. Es gehe, so der DFG-Bericht für 1930/31, um ,die Verteidigung des gegenwärtigen und zukünftigen Raumes für das deutsche Volk'. (Wagner 2010a: 30)

Nach 1945 stellten die Agrarsoziologen „unter stillschweigender Ausblendung der Rassesemantik“ ihre Expertise wie bisher zur Verfügung, ohne sich gedanklich von „Volkstumskategorien“ verabschiedet zu haben (Oberkrome 2010: 429-431).

Die Autoren beschreiben die Bedeutung des Expertenwissens im Nationalsozialismus, die Handlungsfähigkeit, Selbstrekrutierung, Selbstindienstnahme und Initiative der Wissenschaftler, die einem eben nicht wissenschaftsfeindlichen Staat ihre Problemlösungskompetenz andienten, wofür sie materielle, finanzielle wie personelle Ressourcen erhielten. Im Rahmen einer eben nicht orientierungslosen nationalsozialistischen Wissenschaftspolitik habe dieses zu einer nicht zu unterschätzenden Effektivität 
geführt und zwar, ohne Wissenschaftler zu missbrauchen. Die „eigentliche Leistung von DFG und RFR [...] bestand darin, den Hochschulforschern einen Freiraum bereitzustellen, in dem sie ihre Kreativität" und in grundsätzlicher Übereinstimmung mit der herrschenden Politik und im Selbstverständnis eines Wissenschaftlers ihr „Engagement für das Regime und seine Kriegziele“ verwirklichen konnten (Wagner 2010a: 35).

Geisteswissenschaften wies die DFG eine Dienstleitungsfunktion im Rahmen der Wissenschaften zu und etablierte, ausgestattet mit einem feinen Sensorium für noch nicht ausgesprochene gesellschaftliche Erwartungshaltungen, „die außerwissenschaftliche Relevanz eines Projektes von Beginn an als Kriterium des Begutachtungsverfahrens“" (Wagner 2010b: 355 f.).

Mit ihrer Förderpraxis half die DFG sowohl bei der Formierung sich entwickelnder Disziplinen als auch interdisziplinärer Themenfelder, installierte sie sich selbst als Instanz wissenschaftlicher Qualitätssicherung, beeinflusste überkommene Kontinuitäten ebenso wie erforderliche Wandlungen von Forschungsrichtungen und wirkte als Vermittlungsinstanz zwischen innerfachlichen Debatten und außerwissenschaftlichen Diskursen.

Nach 1945 zogen Entnazifizierung und die Unterscheidung von „scheinbar seriösen Wissenschaftlern“ von „völkischen Dilettanten“ keinen tiefgreifenden Wandel in der Personalstruktur der Wissenschaftler nach sich. Vielmehr diente die klare Identifikation in der Gleichung von Nationalsozialismus und Dilettantismus dazu, sich weiterreichender Überlegungen $\mathrm{zu}$ Geschichte, Fragestellungen und Methoden des eigenen Faches zu entledigen, sie in Einzelfällen zu personalisieren und so eine fachinterne Selbstvergewisserung vorzunehmen. Die Kategorien „Volk“, „Gemeinschaft“ und „Führer“ gingen in "Westen“, „Gesellschaft" und „Pluralismus“ auf (Wagner 2010b: 357).

In der 1949 wieder gegründeten DFG rückte nun die „Politikferne“ als wichtiges Qualitätskriterium und so als Grundlage für die Bewilligung von Projekten weit nach vorne und diente der DFG gleichzeitig als Ausweis eigener Seriosität.

An der Ostforschung, die sich über die Systemwechsel hinweg durch ihre Nähe zur Politik auszeichnet, ist ablesbar, wie reibungslos eine selbst so eng mit dem Nationalsozialismus verbundene Forschung sich durch semantische Transformationen an veränderte Bedingungen anpassen konnte, welche Rolle dabei die politischen Rahmenbedingungen spielten, ebenso aber auch, welchen Einfuß Politik zu nehmen in der Lage war.

Nach 1945 ist in der westdeutschen Ostforschung eine starke personelle wie institutionelle und methodische Kontinuität zu beobachten. Durch Aufgabe des radikalen Vokabulars wurden semantische Transformationen vorgenommen, ohne das Selbstverständnis grundlegend zu revidieren. Antibolschewistische Ressentiments gingen im Antikommunismus auf, der als Integrationsideologie der westdeutschen Nachkriegsgesellschaft fungierte und 
das neue Bedrohungsszenario lieferte. Das Wissen der Ostforscher schien von Bedeutung.

Diese ,neue Kampfstellung gegen den Osten' war im Grunde die alte und den Ostforschern sehr vertraut. Viele ihrer Vertreter verharrten in dieser Position, nachdem sie sich auf die Seite des Westens begeben und ihre ehemals imperialen Ziele gegenüber Ostmittel- und Osteuropa in die ,Verteidigung des Abendlandes' umgewidmet hatten. (Unger 2007: 425-431 unter Bezug auf Fellner 1989)

Mit dem Rückenwind des Kalten Krieges, nun zur Verteidigung der westlichen Werte, jetzt mit dem die nationalstaatlichen Grenzen negierenden Konzept vom Abendland, kämpften sie in der alten Frontstellung an einer näher gerückten ,eisernen' Grenze, weiterhin den Blick nach Osten gewandt, diesmal gegen ,totalitäre Regime', ohne diese Kategorie als Erkenntnishilfe zur Befragung der eigenen Geschichte berühren zu wollen. Erst veränderte politische Rahmenbedingungen und gesellschaftliche Wandlungsprozesse sowie die „Forderungen nach einer Demokratisierung der Wissenschaft" seit Mitte der 1960er Jahre und die bewusste Neuausrichtung in der Osteuropapolitik durch Willy Brandt verhalfen ehemaligen Minderheitenpositionen in der Disziplin zum Durchbruch und führten seit Beginn der 1970er Jahre zu einer Transformation der Ostforschung zur Osteuropaforschung ohne nationalistische Vorannahmen (Unger 2007: 437 f.).

\section{Anmerkungen}

$1 \mathrm{Zu}$ diesem Thema wurde von einer Forscherinnengruppe im Auftrag der DFG auch eine Ausstellung erarbeitet, die in Verbindung mit wissenschaftlichen Konferenzen national wie international gezeigt wurde (Heinemann/Oberkrone/Schleiermacher/Wagner 2006).

\section{Literatur}

Eichholtz, Dietrich, 1982. Der „Generalplan Ost“. Über eine Ausgeburt imperialistischer Denkart und Politik (mit Dokumenten). Jahrbuch für Geschichte, 26, 217-274.

Fellner, Fritz, 1989. Nationales und europäisch-atlantisches Geschichtsbild in der Bundesrepublik und im Westen in den Jahren nach dem Ende des Zweiten Weltkrieges. In: Ernst Schulin (unter Mitarbeit von Elisabeth Müller-Luckner), Hg., Deutsche Geschichtswissenschaft nach dem Zweiten Weltkrieg (1945-1965) [= Schriften des Historischen Kollegs: Kolloquien, 14]. München: Oldenbourg, 213-226.

Heinemann, Isabel, 2006. Wissenschaft und Homogenisierungsplanungen für Osteuropa. Konrad Meyer, der "Generalplan Ost" und die Deutsche Forschungsgemeinschaft. In: Isabel Heinemann und Patrick Wagner, Hg., Wissenschaft - Planung - Vertreibung. Neuordnungskonzepte und Umsiedlungspolitik im 20. Jahrhundert [= Beiträge zur Geschichte der Deutschen Forschungsgemeinschaft, 1]. Stuttgart: Steiner, 45-72.

Heinemann, Isabel/Oberkrome, Willi/Schleiermacher, Sabine und Patrick Wagner, 2006. Wissenschaft, Planung, Vertreibung. Der Generalplan Ost der Nationalsozialisten. Katalog zur Ausstellung der Deutschen Forschungsgemeinschaft. Bonn: DFG. 
Herbert, Ulrich, 2010. Der deutsche Professor im Dritten Reich. Vier biografische Skizzen. In: Karin Orth und Willi Oberkrome, Hg., Die Deutsche Forschungsgemeinschaft 1920-1970. Forschungsförderung im Spannungsfeld von Wissenschaft und Politik [= Beiträge zur Geschichte der Deutschen Forschungsgemeinschaft, 4]. Stuttgart: Steiner, 483-502.

Kárný, Miroslav, 1977. Generálnî Plán Východ. Českoslovénsky časopis historický, 25, 345-382.

Oberkrome, Willi, 2010. Agrarische Selbstversorgung und bäuerliche Ordnung. Die deutsche landwirtschaftliche Forschung 1920-1960. In: Karin Orth und Willi Oberkrome, Hg., Die Deutsche Forschungsgemeinschaft 1920-1970. Forschungsförderung im Spannungsfeld von Wissenschaft und Politik. Stuttgart: Steiner, 425-432.

Rössler, Mechtild, 1990. „Wissenschaft und Lebensraum“. Geographische Ostforschung im Nationalsozialismus. Ein Beitrag zur Disziplingeschichte der Geographie [= Hamburger Beiträge zur Wissenschaftsgeschichte, 8]. Berlin, Hamburg: Dietrich Reimer.

Rössler, Mechtild/Schleiermacher, Sabine, 1993. Der „Generalplan Ost“ und die „Modernität“ der Großraumordnung. Eine Einführung. In: Mechtild Rössler und Sabine Schleiermacher, Hg., Der "Generalplan Ost". Hauptlinien der nationalsozialistischen Planungs- und Vernichtungspolitik [= Schriften der Hamburger Stiftung für Sozialgeschichte des 20. Jahrhunderts]. Berlin: Akademie-Verlag, 7-11.

Roth, Karl Heinz, 1985. Bevölkerungspolitik und Zwangsarbeit im „Generalplan Ost“. Mitteilungen der Dokumentationsstelle zur NS-Sozialpolitik, 1, Heft 3, 70-93.

Roth, Karl Heinz, 1993. „Generalplan Ost“ - „Gesamtplan Ost“. Forschungsstand, Quellenprobleme, neue Ergebnisse. In: Mechtild Rössler und Sabine Schleiermacher, Hg., Der "Generalplan Ost". Hauptlinien der nationalsozialistischen Planungs- und Vernichtungspolitik [= Schriften der Hamburger Stiftung für Sozialgeschichte des 20. Jahrhunderts]. Berlin: Akademie-Verlag, 25-95.

Wagner, Patrick, 2010a. „Reservat der Ordinarien“. Zur Geschichte der Deutschen Forschungsgemeinschaft zwischen 1920 und 1970. In: Karin Orth und Willi Oberkrome, Hg., Die Deutsche Forschungsgemeinschaft 1920-1970. Forschungsförderung im Spannungsfeld von Wissenschaft und Politik [= Beiträge zur Geschichte der Deutschen Forschungsgemeinschaft, 4]. Stuttgart: Steiner, 23-38.

Wagner, Patrick, 2010b. Grenzwächter und Grenzgänger der Wissenschaft. Die Deutsche Forschungsgemeinschaft und die Geistes- und Sozialwissenschaften 1920-1970. In: Karin Orth und Willi Oberkrome, Hg., Die Deutsche Forschungsgemeinschaft 1920-1970. Forschungsförderung im Spannungsfeld von Wissenschaft und Politik [= Beiträge zur Geschichte der Deutschen Forschungsgemeinschaft, 4]. Stuttgart: Steiner, 347-361.

\section{Sabine Schleiermacher}

Forschungsschwerpunkt Zeitgeschichte

Institut für Geschichte der Medizin

Charité - Universitätsmedizin Berlin

Luisenstrasse 64/65

10117 Berlin

Deutschland

E-Mail: Sabine.Schleiermacher@charite.de 